\title{
On the theoretical error bound for estimating psychometric functions
}

\author{
Huanping Dai • Virginia M. Richards
}

Published online: 17 November 2010

(C) Psychonomic Society, Inc. 2010

\begin{abstract}
The theoretical limits to the amount of error, or the Cramer-Rao bounds, were derived for estimating psychometric functions. These theoretical error bounds were compared with the variability of psychometric functions estimated from human as well as computer-simulated observers. For the simulated observers, due to the limited efficiency of the sampling strategies, including the placement of the signals and the distribution of the trials, the variances of the estimated parameters are seven times the theoretical bound for threshold and 22 times that for slope. For the human observers, the variance is 18 times the theoretical bounds for threshold and 80 times that for slope. Therefore, a major portion of the variances (60\% for threshold and $73 \%$ for slope) for the human observers is associated with factors other than sampling strategies. Further improvement of the accuracy for estimating psychometric functions will depend on not only optimizing the sampling strategy, but also better understanding the various sources of error related to the behavior of human observers.
\end{abstract}

Keywords Psychometric function - Cramer-Rao bound

\section{Introduction}

The psychometric function (PF) is a classic measure that characterizes an observer's behavior in perceptual tasks

H. Dai $(\square)$

Department of Speech, Language, and Hearing Sciences,

University of Arizona,

1131 E. 2nd Street,

Tucson, AZ 85721, USA

e-mail: hdai@email.arizona.edu

V. M. Richards

Department of Cognitive Sciences, University of California, Irvine, CA 92697, USA (e.g., Green \& Swets, 1966; Macmillan \& Creelman, 2005). The function not only describes the sensitivity of the observer in detecting a weak signal, or a small change along some stimulus dimension, it also can reveal the decision strategy adopted by the observer. Given the rich amount of information contained in PFs, the measurement of such functions is common practice in perception research. In this note we examine the theoretical limit to the accuracy with which PFs can be estimated.

A PF describes the proportion of correct response $(p c)$ in a particular sensory or perception task (e.g., detection of a tone in quiet) as a function of signal strength (e.g., the signal energy), $x$. The values of $p c$ are typically fitted with a standard function. For example, psychoacoustic researchers (e.g., Buus, Schorer, Florentine, \& Zwicker, 1986; Dai, 1995; Egan, Lindner, \& McFadden, 1969) have modeled PFs using a Gaussian cumulative distribution function $\Phi$ with a threshold parameter $\alpha$ and a slope parameter $\beta$, i.e., $p c=\Phi(x, \alpha, \beta)$. For this case, the PF is completely determined once the threshold and slope parameters are known. Thus, the study of PFs reduces to estimating the threshold and slope parameters. Estimating these parameters involves the selection of a set of values of the signal strengths, $x$, and the estimation of an observer's response at the selected signal values. Following psychophysical measurement, a pre-specified probability function, such as a Gaussian cumulative distribution, is fitted to the proportion of correct responses at the signal levels. The parameters are estimated as the values that minimize the differences (e.g., an RMS measure) between the obtained and predicted proportion of correct responses.

The accuracy of PFs estimated with human observers depends on two groups of factors. The first group of factors is related to the sampling strategy, specifically, which signal levels are to be tested, and how the number of trials are to be allocated over the signal levels 
selected. These factors are non-specific to human behavior. The second group includes factors related to human behavior, such as practice and learning, fluctuation in alertness and attention (or lapses), degree of fatigue, effect of uncertainty about the signal parameters, switching among multiple cues while performing the task, any sequential dependence of stimulus presentation and subjective response, among other things.

Optimizing sampling strategy has been a major objective in previous efforts (e.g., Hall, 1968, 1981; Hawley \& Colburn, 1995; Jesteadt, 2005; King-Smith \& Rose, 1997; Klein, 2001; Lam, Dubno, \& Mills, 1999; Leek, 2001; Leek, Hanna, \& Marshall, 1992; McKee, Klein, \& Teller, 1985; Miller \& Ulrich, 2001; O'Regan \& Humbert, 1989; Schlauch \& Rose, 1990; Taylor \& Creelman, 1967; Watt \& Andrews, 1981; Wichmann \& Hill, 2001a, 2001b). The premise is that, given a fixed total number of trials for measuring a PF, of all the possible ways of selecting the signal levels and distributing the trials, there will be one implementation that will produce the most accurate estimate of the parameters, resulting in the least amount of error from the true values of the parameters. How much improvement in the accuracy of estimated PFs can be achieved by optimizing sampling strategy will depend on two measures of relative variability. First, it depends on the variability associated with sampling relative to the within-subject variability typically observed for human observers. One way to separate the variability specific to sampling strategy from the variability specific to the human factors is by examining a computer-simulated observer assuming the same sampling strategy as for the human observers. Second, it depends on the variability associated with the existing sampling strategies relative to the theoretical limit of error. Only if the variability associated with sampling accounts for a major proportion of the total withinsubject variability for human observers, and at the same time far exceeds the theoretical limit, can we expect any significant improvement in the accuracy of estimated PFs by optimizing sampling strategy. Thus, knowing the theoretical limit of error for estimated PFs is of significant importance for both theoretical and practical reasons.

The purpose of this note is to derive the theoretical limit to the accuracy for estimating the parameters of a $\mathrm{PF}$ and to compare the theoretical limit with empirically estimated variability. Here we seek to determine the lower bound for the amount of error that can be achieved in estimating the parameters. In the framework of statistical estimation theory, the lower bound of error is derived as the so-called Cramer-Rao bound (CRB). We begin by deriving the CRB for the threshold and slope parameters for one form of a PF: a Gaussian cumulative distribution function. Then we discuss the error bound for the two parameters, including their dependence on the total number of trials and the interaction between the estimates of the parameters. Finally, using methods consistent with those currently in practice, error sizes for threshold and slope parameters are estimated for both human and computer simulation data sets. The results show that, while the error sizes for both human and simulated observers far exceed the theoretical limit, the variability estimated for the simulated observers explains only a minor portion of that estimated for the human observers. Therefore, improving the accuracy for estimating psychometric functions requires our efforts not just in optimizing the sampling strategy but also in better understanding the behavior of human observers.

This study follows the long tradition of the analysis of ideal observers in the field of psychophysics, for which the reader is referred to other sources of references (e.g., Green \& Swets, 1966; Rouder et al., 2007; Taylor \& Creelman, 1967).

\section{Underlying assumptions for psychometric functions}

In this article, we focus on PFs for a 2-alternative, forced-choice (2AFC) task, for which the values of $p c$ range from $1 / 2$ for no signal (i.e., $x=0$ ) to 1 as the signal strength approaches infinity. For the purpose of analysis, and without loss of generality, we express the PF as the probability of responding ' 2 '. This will be denoted as $p 2$.

Let $X_{1}$ and $X_{2}$ represent the random observation variables for the first and second intervals of the $2 \mathrm{AFC}$ task, and redefine the signal strength as $x=E\left(X_{2}-X_{1}\right)$, where $E(\cdot)$ represents the expectation of the argument within the parenthesis. Defined this way, the values of $p 2$ range from $p 2=0$ as $x \rightarrow-\infty$ (when the signal is added to the 1 st interval) to $p 2=1$ as $x \rightarrow \infty$ (when the signal is added to the 2 nd interval). Note that the expected value of $p 2$ on the positive half of the $x$ axis is identical to the expected value of the original psychometric function, $p c$. Moreover, the expected values of $p 2$ on the negative half of the $x$ axis can be made identical to the expected value of $p c$ via simple transformations of $x^{\prime}=-x$ and $p^{\prime}=1-p 2$. For this reason, the two forms of psychometric functions $p c$ and $p 2$ share the same underlying probability density function. We take advantage of this fact to drive the probability density function as well as the CRB based on $p 2$, but will apply the results of the analysis to the original psychometric function $p c$.

The current analysis is carried out within the framework of the signal-detection theory: The values of $p 2$ 
are fitted using a cumulative Gaussian distribution function, $\Phi\{N(\mu=0, \sigma=1)\}$. First, a sensitivity variable, $\xi$, is introduced. $\xi$ is related to the index of sensitivity, $d$ ', by $d^{\prime}=\sqrt{2}|\xi|$. With these definitions, the $\mathrm{PF}$ for a $2 \mathrm{AFC}$ task can be expressed as

$p 2=\Phi(\xi)$,

and the sensitivity variable $\xi$ is related to the signal strength variable $x$ by

$\xi=\operatorname{sign}(x)\left(\frac{|x|}{\alpha}\right)^{\beta} / \sqrt{2}$,

where $\alpha>0$ is the threshold parameter and $\beta>0$ the slope parameter. It follows that the index of sensitivity is related to the signal strength as follows: $d^{\prime}=\left(\frac{|x|}{\alpha}\right)^{\beta}$. The upper panel of Fig. 1 shows PFs corresponding to three different values of $\beta(0.5,1,2)$ and a fixed value of $\alpha=1$. This form and similar forms of PFs have been used in previous studies (e.g., Buus et al., 1986; Dai, 1994, 1995; Egan et al., 1969; Green \& Swets, 1966; Leek et al., 1992; Schlauch \& Rose, 1990).

Note that in this development the value of $d^{\prime}$ is symmetric relative to the value of $x=0$, i.e., it is assumed that the value of $d$ ' is the same when $p 2=0.8$ and $p 2=0.2$ (both of which are "correct" at an $80 \%$ rate). If the threshold is defined as the signal strength corresponding to $d^{\prime}=1$ (or $p c=76 \%$ ), $\alpha$ represents the threshold. Furthermore, when $d$ ' is plotted as a function of signal level on a logarithmic axis, the resulting psychometric function is a line with a slope of $\beta$.

\section{The Cramer-Rao bound for the threshold and slope parameters}

The derivation of the CRB for threshold and slope estimates is an example of a problem of statistical estimation. Suppose the probability density function of a random variable(s) $\varsigma$, is $\mathrm{h}\left(\varsigma, \lambda_{1}, \lambda_{2}, \ldots\right)$, where $\lambda_{\mathrm{i}}$ are unknown parameters. The values of these parameters can be estimated based on samples of the random variable, $\varsigma$. The best accuracy for estimating the parameters depends on the sample size as well as the particular form of the density function. For such problems, Cramer, in his now classic volume, Mathematical Methods of Statistics (1946), provided the formula for deriving the theoretical lower bound of error for parameter estimation. This lower bound of error is referred to as the Cramer-Rao bound in the literature of statistical estimation.

For PFs, suppose the underlying probability density function is $\mathrm{g}(x, \alpha, \beta)$, with both $\alpha$ and $\beta$ being real, positive numbers. Using Eqs. 1 and 2, the form of $\mathrm{g}(x, \alpha, \beta)$ is derived in the Appendix; it is

$g(x, \alpha, \beta)=\frac{1}{2 \sqrt{\pi}}\left(\frac{\beta}{\alpha}\right)\left(\frac{|x|}{\alpha}\right)^{\beta-1} e^{-\frac{1}{4}\left(\frac{|x|}{\alpha}\right)^{2 \beta}}$.

The lower panel of Fig. 1 shows the shape of the density function corresponding to the same parameters used for generating the PFs in the upper panel. Owing to its symmetry about zero, the density function, although derived from $p 2$, underlies the original PF $p c$. Thus, the form of the probability density function evaluated in the
Fig. 1 Psychometric functions (upper panel) and the underlying probability density functions (lower panel) corresponding to a threshold parameter of $\alpha=1$ and slope parameters of $\beta=0.5$ (dashed lines), $\beta=1$ (dark solid lines, which represent Gaussian functions), and $\beta=2$ (grey lines)
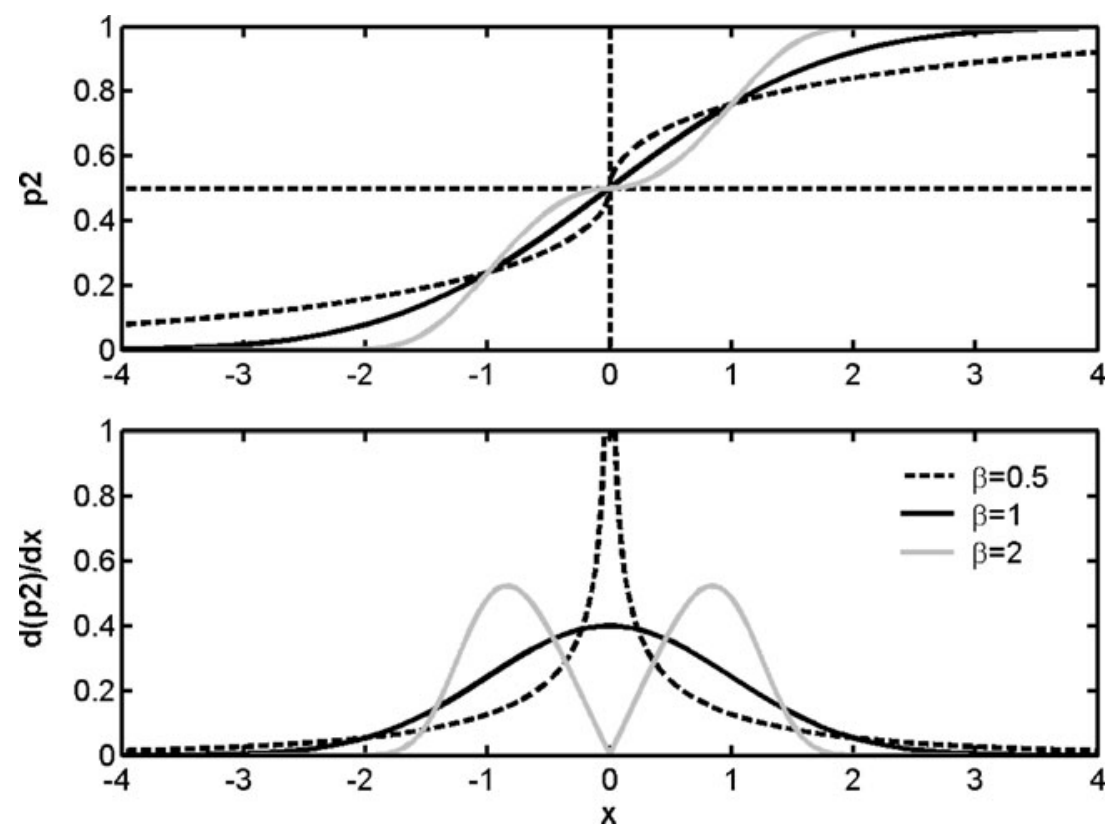
following is as Eq. 3 except the signal strength, $x$, ranges from 0 to $\infty$. That is, $p 2$ will no longer be considered, and the discussion will focus on $p c$ (i.e., the absolute value terms in equations above are removed because the original signal strength, which ranges from 0 to infinity, is used).

According to Cramer (1946), the variances for estimating two unknown parameters are bounded by the following expressions:

$$
V_{\alpha}^{C R B}=\frac{1}{n \Delta} E\left(\frac{\partial \log (g)}{\partial \beta}\right)^{2}
$$

and

$$
V_{\beta}^{C R B}=\frac{1}{n \Delta} E\left(\frac{\partial \log (g)}{\partial \alpha}\right)^{2}
$$

where $E(\cdot)$ represents the expected value of the quantity within the parenthesis, $n$ is the sample size (i.e., the total number of trials used for estimating the PF), and

$\Delta=E\left(\frac{\partial \log (g)}{\partial \alpha}\right)^{2} E\left(\frac{\partial \log (g)}{\partial \beta}\right)^{2}-E^{2}\left(\frac{\partial \log (g)}{\partial \alpha} \frac{\partial \log (g)}{\partial \beta}\right)$.

The lower bound of the standard deviations for estimating the two parameters $\left(\sigma_{\alpha}^{C R B}=\sqrt{V_{\alpha}^{C R B}}\right.$ and $\left.\sigma_{\beta}^{C R B}=\sqrt{V_{\beta}^{C R B}}\right)$ can be obtained from Eqs. 3 to 6 , although in Eq. 3 the absolute value is removed. Using numerical methods, we obtained $\sigma_{\alpha}^{C R B}$ and $\sigma_{\beta}^{C R B}$ for threshold and slope values ranging $4 \geq \alpha \geq 0.25$ and $4 \geq \beta \geq 0.25$ (MATLAB code is included in the supplementary material available on-line). The range for the threshold parameter may seem narrow, but as will be shown below, the relative error bound for $\alpha, \sigma_{\alpha}^{C R B} / \alpha$ and the error bound for $\beta, \sigma_{\beta}^{C R B}$ are independent of the value of $\alpha$. Thus, the choice of the range for $\alpha$ is largely inconsequential. In contrast, the error bound for $\beta$, either in absolute or relative error, does depend on the value of $\beta$. Our choice of the range for $\beta$ in the current analysis covers the slope values typically encountered in the psychophysical literature (e.g., Laming, 1986).

Figure 2 illustrates the sizes of $\sigma_{\alpha}^{C R B}$ and $\sigma_{\beta}^{C R B}$ for a range of sample sizes, $n$ (i.e., the number of trials assumed in estimating a PF) for $\alpha=1$ and $\beta=1$. As implied by Eqs. 4 and 5, both $\sigma_{\alpha}^{C R B}$ and $\sigma_{\beta}^{C R B}$ decrease in proportion to the inverse of $\sqrt{n}$. Because $\alpha=1$ and $\beta=1, \sigma_{\alpha}^{C R B}$ and $\sigma_{\beta}^{C R B}$ also reflect the sizes of the relative error bounds for the two parameters (i.e., $\sigma_{\alpha}^{C R B} / \alpha$ and $\sigma_{\beta}^{C R B} / \beta$ ). In this case, the two relative error bounds for $\alpha$ and $\beta$ have similar values. For example, for a sample size of $n=400$, we have $\left.\sigma_{\alpha}^{C R B}\right|_{n=400} / \alpha \approx 0.05$ and $\left.\sigma_{\beta}^{C R B}\right|_{n=400} / \beta \approx 0.04$.

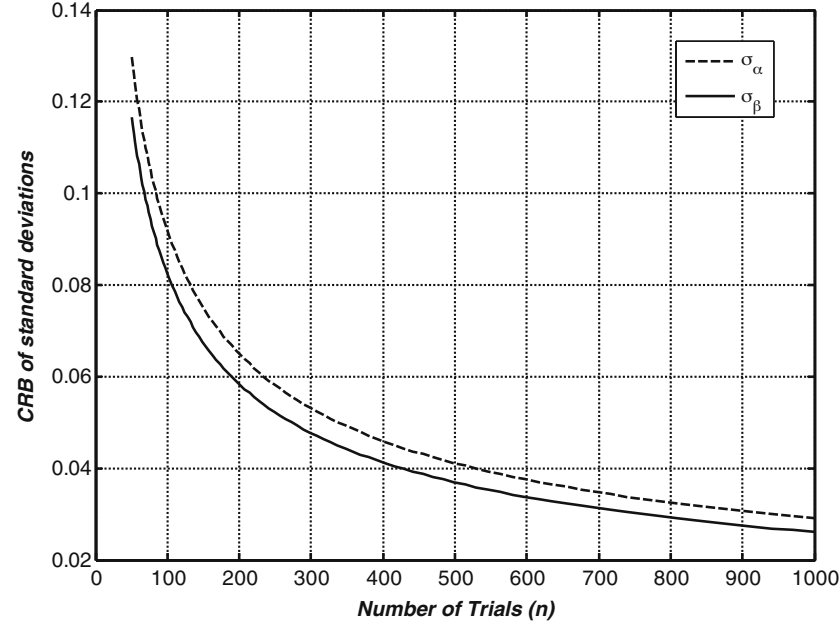

Fig. 2 Cramer-Rao bounds in standard deviation $\left(\sigma_{\alpha}^{C R B}\right.$, and $\left.\sigma_{\beta}^{C R B}\right)$ for threshold (solid line) and slope (dashed line) as a function of sample size $(n)$. Both parameters are set to unity (i.e., $\alpha=1$ and $\beta=1$ ) in deriving the standard deviations

Figure 3 presents the relative error bound (left panel for threshold and right panel for slope) as a joint function of $\alpha$ and $\beta$. We scaled the standard deviations by the square root of the sample size (i.e., $\left.\sigma_{\alpha}^{C R B}\right|_{n=1}=\sqrt{n} \sigma_{\alpha}^{C R B}=\sqrt{n V_{\alpha}^{C R B}}$, and $\left.\sigma_{\beta}^{C R B}\right|_{n=1}=\sqrt{n} \sigma_{\beta}^{C R B}=\sqrt{n V_{\beta}^{C R B}}$ ) to allow a presentation of the results without regard to number of trials. The scaled standard deviations $\left.\sigma_{\alpha}^{C R B}\right|_{n=1}$ and $\left.\sigma_{\beta}^{C R B}\right|_{n=1}$ may be regarded as equivalent to the error bounds based on a single sample (i.e., $n=1$ ).

The results show three main trends. First, the relative error bounds for both threshold and slope parameters are independent of the value of the threshold parameter $\alpha$. Second, the relative error bound for threshold estimate $\left(\left.\sigma_{\alpha}^{C R B}\right|_{n=1} / \alpha\right.$, left panel) depends on the value of the slope, $\beta$. It is highest when the slope is shallowest at $\beta=0.25$, and is reduced by a factor of approximately 20 when the slope is increased to $\beta=4$. This trend is consistent with the idea that the standard deviation of proportion of correct $(p c)$ score relates to the standard deviation of the estimated threshold via the slope of the psychometric function (e.g., Green, 1990). Given that $p c=0.76$ corresponds to the signal strength at threshold, $x=\alpha$, on the PF, the standard deviation for estimating $p c$ is $\sigma_{p c}=\sqrt{\frac{p c(1-p c)}{N}}$ (based on the binomial distribution), in which $N$ is the number of trials for estimating the value of $p c$. Since the variability of $p c$, i.e., $\sigma_{p c}$, is fixed (for given $p c$ and $N$ ), a steeper PF is expected to project the fixed $\sigma_{p c}$ onto a smaller error size for estimating the threshold. Thus, both the error size and the error bound for estimating the threshold reduce as the PF steepens.

The third main trend is that the relative error bound for slope estimate $\left(\left.\sigma_{\beta}^{C R B}\right|_{n=1} / \beta\right.$, right panel $)$ is independent of the value of the slope when $\beta \geq 1$, but increases slightly (by 
Fig. 3 Relative error bounds for threshold $\left(\left.\sigma_{\alpha}^{C R B}\right|_{n=1} / \alpha\right.$, left panel, a) and slope $\left(\left.\sigma_{\beta}^{C R B}\right|_{n=1} / \beta\right.$, right panel, b) as a joint function of threshold and slope. Note the different scales of the $\mathrm{z}$-axes for the two panels a

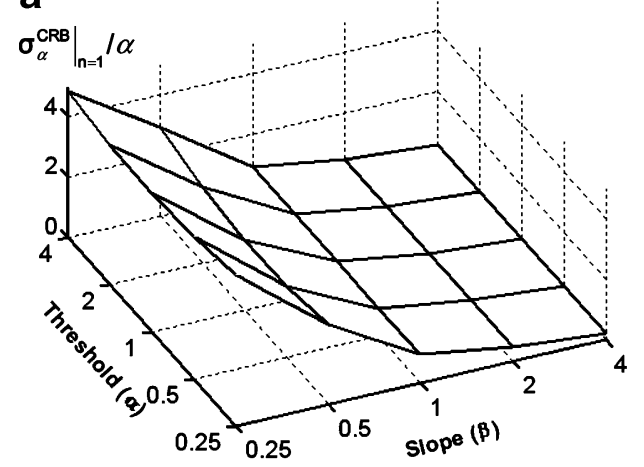

b

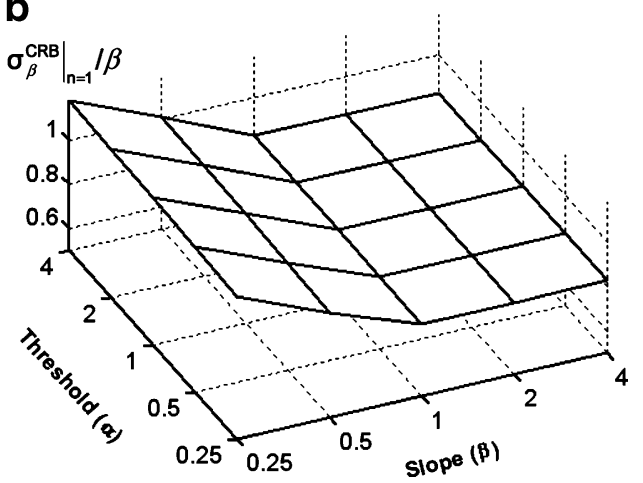

a factor of $\sim 1.4$ at $\beta=0.25$ ) with decreasing slope when $\beta<1$. The reasons for this effect remain unclear. We cannot rule out the possibility that the error bounds computed at small values of $\beta$ were prone to artifacts in deriving the numerical solutions. Simulation data presented below suggest this is a likely account of this result.

\section{Comparison of the Cramer-Rao bound and estimated errors from human and computer-simulated observers}

\section{A. Human observers}

In this section we present one example to illustrate the variability of PFs estimated from human observers. In a previous study (Dai, 1995), PFs of two subjects performing auditory frequency discrimination were measured multiple times with the specific purpose of evaluating the variability. The results from that study are re-considered here for comparison with the derived CRBs and the variability from the simulated observer. The measurements involved three psychophysical procedures (an adaptive method with either a 2-down-1-up or a 3-down-1-up rule, and the constantstimulus method; for a description of these procedures, the reader is referred to Macmillan \& Creelman, 2005).

For one observer a total of 15 PFs were fitted (5 for each psychophysical procedure) and for the other observer a total of 12 PFs were fitted (4 for each psychophysical procedure). Each fitted PF was based on a total of 900 trials. The estimate of the standard error of the estimated "human" threshold $\left(\alpha^{\text {Human }}\right)$ and "human" slope $\left(\beta^{\text {Human }}\right)$ parameters were based on 5 and 4 replicates, depending on the observer. The estimates of the standard errors did not depend strongly on the psychophysical method tested, so the data from the three different psychophysical methods were averaged for each observer. The next step was to estimate the ratios, $\sigma_{\alpha}^{\text {Human }} / \alpha^{\text {Human }}$ and $\sigma_{\beta}^{\text {Human }} / \beta^{\text {Human }}$ for each observer. Then, the ratios were averaged across observers. The average relative error is $\sigma_{\alpha}^{\text {Human }} / \alpha^{\text {Human }}=$
0.19 for threshold and $\sigma_{\beta}^{\text {Human }} / \beta^{\text {Human }}=0.25$ for slope. ${ }^{1}$ The error size is about four times the CRB for threshold ( 0.19 vs 0.05$)$ and nine times that for slope (0.25 vs 0.028$)$. Consequently, the variances from the human observers are 14 times the CRB for threshold and 80 times that for slope.

\section{B. Computer-simulated observers}

The main purpose of the following analysis is to compare the variability of the estimated parameters for simulated observers with that of the human observers and the CRBs. In addition, we are interested in whether the variability of the simulated PFs follows trends similar to those of the CRBs. To address these issues, PFs were generated via Monte Carlo simulation over the same parameter space explored in the analysis of the CRB (i.e., $4 \geq \alpha^{\text {Simulated }} \geq 0.25$ and $4 \geq \beta^{\text {Simulated }} \geq 0.25$ ). The MATLAB code used for the simulation is included in the supplementary material available on-line.

The method of constant stimuli was used. Five signal levels that were expected to produce values of $d^{\prime}=0.15$, $0.30,0.60,1.21$, and 2.45 , corresponding to $p c=0.542$, $0.584,0.665,0.805$, and 0.958, were evaluated. Each simulated function had 900 trials, with 180 trials per signal level. This sampling strategy was chosen to approximate the strategy used for the two human subjects described above. For each combination of the generating values of $\alpha^{\text {Simulated }}$ and $\beta^{\text {Simulated }}, 1000$ PFs were simulated and then fitted. Finally, means and standard errors were calculated from the estimates of the threshold and slope. The means of

\footnotetext{
${ }^{1}$ The relative errors of the human observers for estimated thresholds and slopes were derived based on information from Table II of Dai (1995). Let $r$ denote the numbers inside the brackets in that table; these numbers represent $r=\frac{M+s}{M}$, in which $M$ represents the mean (either for threshold or slope) and $s$ the standard error. Thus, the relative error can be obtained as $\frac{s}{M}=r-1$. The relative error averaged over the three psychophysical procedures and the two observers (six values) is 0.19 for the thresholds $\left(\sigma_{\alpha} / \alpha\right)$ and 0.25 for the slopes $\left(\sigma_{\beta} / \beta\right)$. The original 12 values of $r$ are available on-line in the supplementary material of the current article.
} 
Fig. 4 Relative errors of simulated observers for threshold $\left(\sigma_{\alpha}^{\text {Simulated }} / \alpha^{\text {Simulated }}\right.$, left panel, a) and slope $\left(\sigma_{\beta}^{\text {Simulated }} / \beta^{\text {Simulated }}\right.$, right panel, b) ${ }^{\beta}$ as a joint function of threshold and slope a

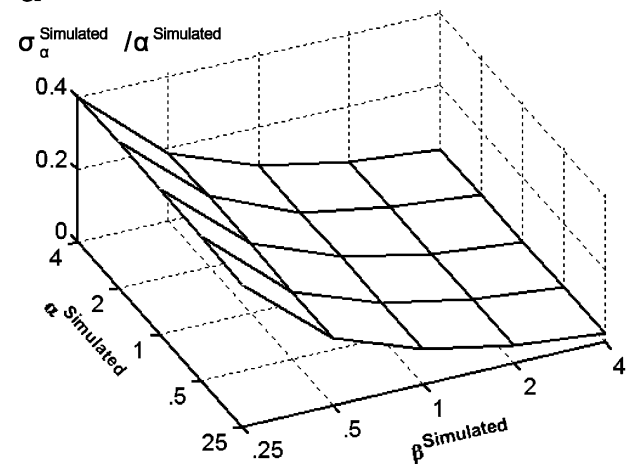

b

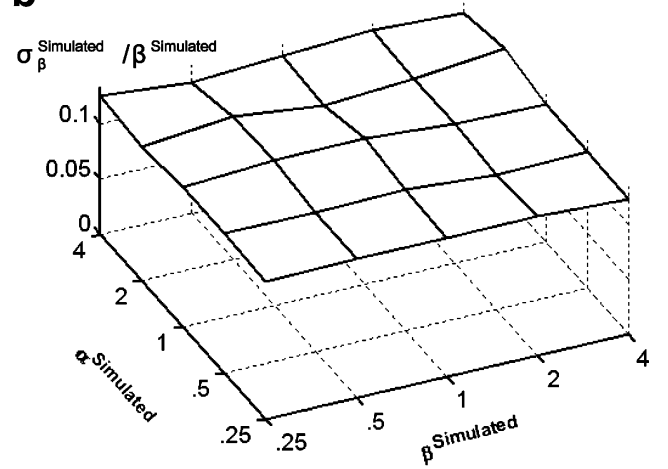

the estimated $\alpha^{\text {Simulated }}$ and $\beta^{\text {Simulated }}$ well matched their true (generating) values, consistent with previous observations (e.g., Dai, 1995) that with $n=900$ trials the bias is negligible.

Figure 4 presents the relative error for the estimated threshold $\left(\sigma_{\alpha}^{\text {Simulated }} / \alpha^{\text {Simulated }}\right.$, left panel $)$ and slope $\left(\sigma_{\beta}^{\text {Simulated }} / \beta^{\text {Simulated }}\right.$, right panel $)$ as a joint function of the true values of $\alpha^{\text {Simulated }}$ and $\beta^{\text {Simulated }}$, in the same format as Fig. 3 for the CRBs. Like the results for the CRBs, the relative error for the threshold does not depend on the value of $\alpha^{\text {Simulated }}$, but does depend on the value of $\beta^{\text {Simulated }}$, which becomes smaller for steeper slopes. The relative error for the slope, however, does not depend on either parameter (right panel, B), remaining nearly equal for all conditions. This finding is slightly different from the CRB results showing somewhat elevated error at small values of slope (right panel of Fig. 3). In light of this finding, the trend of higher CRBs at small values of slope is likely to reflect the limited precision of our numerical solutions of the CRBs.

Figure 5 highlights the differences and similarities between the errors estimated using simulations and the CRBs for the threshold parameter. The estimated standard deviation (dashed line) shows very similar dependence on slope to that

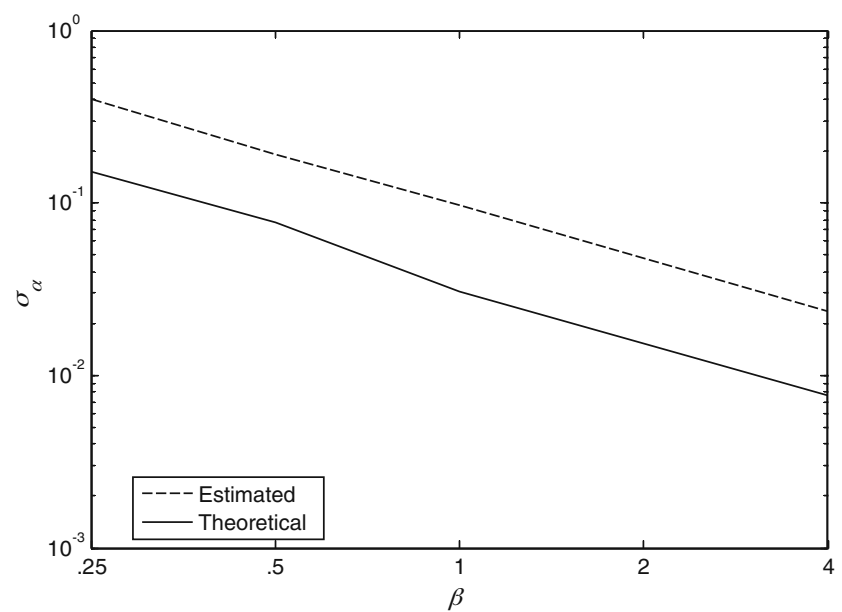

Fig. 5 Estimated (dashed line) standard deviations and the theoretical error bound (solid line) for threshold as a function of slope for the CRB (solid line, calculated for $n=900$ ). Most important, over the range of the values explored for the slope parameter, the standard deviations for estimating the threshold parameter are about three times the theoretical limits.

Further simulations were carried out to evaluate the effect of changes in sample size. The true values of the parameters were fixed at $\alpha^{\text {Simulated }}=1$ and $\beta^{\text {Simulated }}=1$. For both threshold and slope parameters, the estimated error size from the simulated observers decreased in proportion to the inverse of $\sqrt{n}$, much as predicted from the CRB analysis. The estimated error size is about three times the CRB for threshold, and five times the CRB for slope. Therefore, although the absolute accuracy suffers for small sample sizes as expected, the relative efficiency for estimating the parameters of PFs appears to be independent of the sample size.

Finally, the results obtained from the human and simulated observers, along with the CRBs for comparable conditions, are presented together in Table 1. In particular, to be comparable to the human data, the CRBs and simulated results were obtained for the mean slope value from the human subjects $(\bar{\beta}=0.78)$. On the one hand, the standard errors of the simulated observers are a little more than half those of the human observers for both the threshold and slope parameters. Specifically, about $30 \%$ (slope) to $40 \%$ (threshold) of the total variance from the human subjects is associated with the sampling strategy. The remaining variability appears to depend on error sources specific to the behavior of human observers. On the other hand, the variances of the simulated observers are about seven times the CRB for threshold, and 22 times the CRB for slope, suggesting that the sampling

Table 1 Relative errors for estimating the threshold $(\alpha)$ and slope $(\beta)$ parameters from human and simulated observers, and the theoretical limit (Cramer-Rao bound), for psychometric functions estimated with $n=900$ trials

\begin{tabular}{llll}
\hline & Human observers & Simulated observers & CRB \\
\hline$\sigma_{\alpha} / \alpha$ & 0.19 & 0.12 & 0.045 \\
$\sigma_{\beta} / \beta$ & 0.25 & 0.13 & 0.028 \\
\hline
\end{tabular}


strategy applied in the simulation is relatively inefficient. Results obtained by Wichmann and Hill (2001b) exhibited somewhat larger error sizes. Using bootstrap methods, they compared the variability of the estimates for a number of sampling strategies. One of their best strategies (s7) produced a relative error size of $\sigma_{\alpha}^{W \& H} / \alpha^{W \& H}=0.05$ for threshold and $\sigma_{\beta}^{W \& H} / \beta^{W \& H}=0.22$ for slope under the condition of $\alpha^{W \& H}=10, \beta^{W \& H}=3$, and $n=480$ (their Fig. 1). For these parameters, the CRB is 0.016 for threshold and 0.038 for slope. Therefore, the estimated variances are about ten times the CRB for threshold, and 34 times the CRB for slope.

Simulations (e.g., Dai, 1995; Wichmann \& Hill, 2001a, 2001b) have demonstrated clearly that some sampling strategies are more efficient than others. Given the wide gap that still exists between the CRB and the variability due to the sub-optimal efficiency of the currently available sampling strategies, one might expect the accuracy for measuring PFs to improve by optimizing sampling strategy. However, since the variability due to sampling explains only a fraction of the total variability for a human observer, optimizing sampling strategy by itself will be of limited benefit. Significantly improving the accuracy for estimating the PF requires efforts to optimize sampling strategy as well as understand the various sources of error related to human factors. Ultimately, the amount of information that can be extracted from empirically estimated psychometric functions will depend on our ability to assess, monitor, control, and even reduce both sources of error.

\section{Conclusion}

The theoretical limits, or the Cramer-Rao bounds, for the variability of estimated psychometric functions, are a small fraction of the variability observed for human observers. Some of the variability from human observers is associated with the limited efficiency of the existing sampling strategies of signal placement and trial distributions, as revealed by the analysis of computer simulated observers assuming similar sampling strategies as those used by human observers. Most of the variability, however, comes from sources of error specific to the human observers. Therefore, further improvement of the accuracy for measuring psychometric functions will depend on optimizing the sampling strategy as well as better understanding the various sources of error specific to human behavior.

Acknowledgments We gratefully acknowledge the helpful comments and suggestions provided by Dr. C. Douglas Creelman, Dr. Simon Grondin, Dr. Neil Macmillan, and two anonymous reviewers. The paper also benefited from editing by Eva Maria Carreira. This work was supported by University of Arizona and NIH/NIDCD grants R29 DC01827 (HD) and R01 DC02012 (VMR).

\section{Appendix}

In this section we derive the probability density function underlying the psychometric function. Given that a Gaussian cumulative distribution function is fitted to the probability of responding to ' 2 ' 'in a $2 \mathrm{AFC}$ task (Eq. 1), and the relation between the sensitivity variable $\xi$ and the signal strength $x$ (Eq. 2), the probability density function of $x[\mathrm{~g}(x, \alpha, \beta)]$ is related to the Gaussian probability density function of $\xi$ [i.e., $f(\xi)=\frac{1}{\sqrt{2 \pi}} e^{-\xi^{2} / 2}$ ] as follows

$g(x, \alpha, \beta)=f[\xi(x)]\left|\xi^{\prime}(x)\right|$

in which the derivative $\xi^{\prime}(x)$ is the inverse of the Jacobian transformation between the two random variables (e.g., Freeman, 1963); it is

$\xi^{\prime}(x)=\frac{1}{\sqrt{2}} \operatorname{sign}(-x)\left(\frac{\beta}{\alpha}\right)\left(\frac{|x|}{\alpha}\right)^{\beta-1}$.

Plugging Eq. A2 and the expression of $f(\xi)$ into Eq. A1, we obtain

$g(x, \alpha, \beta)=\frac{1}{2 \sqrt{\pi}}\left(\frac{\beta}{\alpha}\right)\left(\frac{|x|}{\alpha}\right)^{\beta-1} e^{-\frac{1}{4}\left(\frac{\mid x}{\alpha}\right)^{2 \beta}}$.

Eq. A3 is referred to as Eq. 3 in the text. Note that, in the special case of $\alpha=\beta=1, g(x, \alpha, \beta)$ becomes the Gaussian probability density function $N(\mu=0, \sigma=\sqrt{2})$, shown as the solid curve in the lower panel of Fig. 1.

\section{References}

Buus, S., Schorer, E., Florentine, M., \& Zwicker, E. (1986). Decision rules in detection of simple and complex tones. The Journal of the Acoustical Society of America, 80, 1646-1657.

Cramer, H. (1946). Mathematical methods of statistics. Princeton: Princeton University Press.

Dai, H. (1994). Signal-frequency uncertainty in spectral-shape discrimination: Psychometric functions. The Journal of the Acoustical Society of America, 96, 1388-1396.

Dai, H. (1995). On measuring psychometric functions: A comparison of the constant-stimulus and adaptive up-down methods. The Journal of the Acoustical Society of America, 98, 3135-3139.

Egan, J. P., Lindner, W. A., \& McFadden, D. (1969). Masking-level differences and the form of the psychometric function. Perception \& Psychophysics, 6, 209-215.

Freeman, H. A. (1963). Introduction to statistical inference. Reading: Addison Wesley.

Green, D. M. (1990). Stimulus selection in adaptive psychophysical procedures. The Journal of the Acoustical Society of America, 87, 2662-2674.

Green, D. M., \& Swets, J. A. (1966). Signal detection theory and psychophysics. Los Altos: Peninsula.

Hall, J. L. (1968). Maximum-likelihood sequential procedure for estimation of psychometric functions. The Journal of the Acoustical Society of America, 44, 370-370. 
Hall, J. L. (1981). Hybrid adaptive procedure for estimation of psychometric functions. The Journal of the Acoustical Society of America, 69, 1763-1769.

Hawley, M.L., \& Colburn, H.S. (1995). Application of confidence intervals and joint confidence regions to the estimation of psychometric functions. Journal of the Acoustical Society of America, 97, 3277(A).

Jesteadt, W. (2005). The variance of d' estimates obtained in yes-no and two-interval forced choice procedures. Perception \& Psychophysics, 67, 72-80.

King-Smith, P. E., \& Rose, D. (1997). Principles of an adaptive method for measuring the slope of the psychometric function. Vision Research, 37, 1595-1604.

Klein, S. A. (2001). Measuring, estimating, and understanding the psychometric function: A commentary. Perception \& Psychophysics, 63(8), 1421-1455.

Lam, C. F., Dubno, J. R., \& Mills, J. H. (1999). Determination of optimal data placement for psychometric function estimation: A computer simulation. The Journal of the Acoustical Society of America, 106, 1969-1976.

Laming, D. (1986). Sensory analysis. London: Academic.

Leek, M. R. (2001). Adaptive procedures in psychophysical research. Perception \& Psychophysics, 63, 1279-1292.

Leek, M. R., Hanna, T. E., \& Marshall, L. (1992). Estimation of psychometric functions from adaptive tracking procedures. Perception \& Psychophysics, 51, 247-256.

Macmillan, N. A., \& Creelman, C. D. (2005). Detection theory: A user's guide (2nd ed.). Cambridge: Cambridge University Press.
McKee, S. P., Klein, S. A., \& Teller, D. A. (1985). Statistical properties of forced-choice psychometric functions: Implications of probit analysis. Perception \& Psychophysics, 37, 286-298.

Miller, J., \& Ulrich, R. (2001). On the analysis of psychometric functions: The Spearman-Kärber Method. Perception \& Psychophysics, 63, 1399-1420.

O’Regan, J. K., \& Humbert, R. (1989). Estimating psychometric functions in forced-choice situations: Significant biases found in threshold and slope estimations when small samples are used. Perception \& Psychophysics, 46, 434-442.

Rouder, J. N., Lu, J., Sun, D., Speckman, P. L., Morey, R. D., \& NavehBenjamin, M. (2007). Signal detection models with random participant and item effects. Psychometrika, 72, 621-642.

Schlauch, R. S., \& Rose, R. M. (1990). Two-, three-, and four-interval forced-choice staircase procedure: Estimator bias and efficiency. The Journal of the Acoustical Society of America, 88, 732-740.

Taylor, M.M., \& Creelman, C.D. (1967). PEST: Efficient estimates on probability functions. Journal of the Acoustical Society of America, 782-787

Watt, R. J., \& Andrews, D. P. (1981). A.P.E: Adaptive probit estimation of psychometric functions. Current Psychological Review, 1, 205-214.

Wichmann, F. A., \& Hill, N. J. (2001a). The psychometric function I: Fitting, sampling, and goodness-of-fit. Perception \& Psychophysics, 63, 1293-1313.

Wichmann, F. A., \& Hill, N. J. (2001b). The psychometric function II: Bootstrap based confidence intervals and sampling. Perception \& Psychophysics, 63, 1314-1329. 